\title{
Systematical Approach to the Exact Solution of the Dirac Equation for A Special Form of the Woods-Saxon Potential
}

\author{
Cüneyt Berkdemir††, Ayşe Berkdemir $\uparrow \oint$ and Ramazan Sever $\ddagger$ \\ $\dagger$ Department of Physics, Faculty of Arts and Sciences, Erciyes University, Kayseri, \\ 38039, Turkey \\ $\ddagger$ Department of Physics, Middle East Technical University, Ankara, 06531, Turkey
}

\begin{abstract}
.
Exact solution of the Dirac equation for a special form of the Woods-Saxon potential is obtained for the s-states. The energy eigenvalues and two-component spinor wave functions are derived by using a systematical method which is called as NikiforovUvarov. It is seen that the energy eigenvalues strongly depend on the potential parameters. In addition, it is also shown that the non-relativistic limit can be reached easily and directly.
\end{abstract}

PACS numbers: 03.65Ge, 03.65Pm, 02.30Gp, 31.30Jv

Submitted to: J. Phys. A: Math. Gen.

$\dagger$ (berkdemir@erciyes.edu.tr)

$\S$ (arsland@erciyes.edu.tr)

$\ddagger$ (sever@metu.edu.tr) 


\section{Introduction}

The Woods-Saxon potential and its various modifications have been received for much interest describing metallic clusters in a successful way. It is used in the central part of the interaction neutron with one heavy-ion nucleus and also for the optical potential model [1]. Differential cross-section in the ${ }^{16} \mathrm{O}+{ }^{12} \mathrm{C}$ elastic scattering are analyzed in some energies by using this potential [2]. The quantum behavior of the relativistic particle in the presence of a central Woods-Saxon potential has settled the possible existence of bound state spectra. The relativistic Dirac-oscillator and Dirac-exponentialtype potential problems have already been established by adding an off-diagonal linear radial term to the Dirac operator for a long time ago [3, 4]. Recently, the relativistic bound states spectrum and its eigenfunctions for the triaxial and axially deformed harmonic oscillators have been derived as well [5]. In addition, a mixture of the Dirac oscillator (tensor potential) with vector and scalar harmonic oscillator potentials has been solved analytically for the general case [6].

Furthermore, only a few articles for the relativistic problems have been written on the Dirac equation with the exponential-type potential. The Dirac equation has been solved by making use of two-component spinors for the exponential type potentials such as Woods-Saxon and Hulthén potentials for a special case. Kennedy has studied the generalized approach to the Woods-Saxon potential and obtained the scattering and bound-state solutions of the one-dimensional Dirac equation. However, more realistic cases have not been discussed [7]. A. D. Alhaidari has just introduced a new formalism to the definition of the radial Dirac equation and solved for a class of shape-invariant potentials [8, 9, 10]. The main point in the formalism is that two coupled first order differential equations resulting from the radial Dirac equation generate Schrödinger-like equations for the two spinor components. Following the procedure given in Ref. [8], we present a new systematical approach to solve the Dirac-Woods-Saxon problem by means of the Nikiforov-Uvarov (NU) method 11. The non-relativistic limit reproduces the well-known non-relativistic energy spectrum and results in the Schrödinger equation for a special form of the Woods-Saxon potential [12].

The article is structured as follows: In Section 2, we briefly introduce an overview of the technical details of the formalism improved by Alhaidari. After that, the basic concepts of the Nikiforov-Uvarov method are given in the same section to solve the Dirac-Woods-Saxon problem. Section 3 is devoted to the solution of the problem to obtain the energy eigenvalues and eigenfunctions by applying the NU method. The paper is concluded with a short summary in Section 4.

\section{Formalism and Method}

We shall first introduce the Alhaidari's formalism proposed to solve the Dirac equation for spherically symmetric potential interactions. Later, the fundamental mathematical relations of the NU method will be summarized to obtain the solution of the Schrödinger- 
like equations easily and systematically.

\subsection{Overview of the Formalism}

Alhaidari's formalism is based on the consideration by writing the relativistic Hamiltonian for a Dirac spinor coupled to a four-component electromagnetic potential $\left(A_{0}, \vec{A}\right)$. After using Gauge invariance and spherically symmetric of the electrostatic potential, free Dirac equation transforms to the matrix representation of the Dirac Hamiltonian (see Ref. 8 for more detail). For convenience, atomic units are selected as $m=e=\hbar=1$ and the speed of light $c$ is matched with $\alpha^{-1}$. Thus, the Hamiltonian for a Dirac spinor in four-component electromagnetic potential $\left(A_{0}, \vec{A}\right)$ can be written as follows:

$$
H=\left(\begin{array}{cc}
1+\alpha A_{0} & -i \alpha \vec{\sigma} \cdot \vec{\nabla}+i \alpha \vec{\sigma} \cdot \vec{A} \\
-i \alpha \vec{\sigma} \cdot \vec{\nabla}-i \alpha \vec{\sigma} \cdot \vec{A} & -1+\alpha A_{0}
\end{array}\right)
$$

where $\alpha$ is the fine structure parameter and $\vec{\sigma}$ are the three $2 x 2$ Pauli spin matrices. Taking the spherically symmetric case and writing $\left(A_{0}, \vec{A}\right)$ as $(\alpha V(r), \hat{r} W(r))$, the twocomponent Dirac equation is obtained as

$$
H=\left(\begin{array}{cc}
1+\alpha^{2} V(r)-E_{R} & \alpha\left[\frac{\kappa}{r}+W(r)-\frac{d}{d r}\right] \\
\alpha\left[\frac{\kappa}{r}+W(r)+\frac{d}{d r}\right] & -1+\alpha^{2} V(r)-E_{R}
\end{array}\right)\left(\begin{array}{c}
g(r) \\
f(r)
\end{array}\right)=0
$$

where $f(r)$ and $g(r)$ are real radial square integrable functions, $E_{R}$ is the relativistic energy and $\kappa$ is the spin-orbit coupling parameter defined as $\kappa= \pm(j+1 / 2)= \pm 1, \pm 2, \ldots$ for $l=j \pm 1 / 2$. However, the current problem is analytically solvable only for $\ell=0$ (sstates). In addition, $V(r)$ and $W(r)$ are the even and odd components of the relativistic potential, respectively. For a given value of the spin-orbit coupling parameter $\kappa$, Schrödinger-like requirement relates the two potential functions as: $W(r)=\frac{1}{\xi} V(r)-\frac{\kappa}{r}$, where $\xi$ is a real parameter and $V(r)$ is not depend on the $\kappa$ parameter. In order to obtain the Schrödinger-like equation in the formalism proposed by A. D. Alhaidari, it is used a global unitary transformation which eliminates the first derivative. Thus $\mathcal{U}(\eta)=\exp \left(\frac{i}{2} \alpha \eta \sigma_{2}\right)$ is applied in Eq.(2). Here, $\eta$ is a real constant and $\sigma_{2}$ is the $2 \times 2$ Pauli matrix which defines the two radial spinor components in terms of the other,

$$
\phi^{\mp}(r)=\frac{\alpha}{C \pm E_{R}}\left[-\xi \pm \frac{C}{\xi} V(r)+\frac{d}{d r}\right] \phi^{ \pm}(r),
$$

with $C=\cos (\alpha \eta)=\sqrt{1-(\alpha \xi)^{2}}>0$,

$$
\left(\begin{array}{l}
\phi^{+}(r) \\
\phi^{-}(r)
\end{array}\right)=\mathcal{U}\left(\begin{array}{c}
g(r) \\
f(r)
\end{array}\right) .
$$

Here $\phi^{ \pm}(r)$ is the upper or lower spinor components respectively. It is emphasized that Eq.(3) with the top and bottom signs are not valid for negative and positive energy solutions respectively. The top and bottom signs in front of $E_{R}$ in Eq.(3) are not 
Systematical Approach to the Exact Solution of the Dirac Equation...

allowed to take the values $-C$ and $+C$. Because these two values are elements of the negative and positive energy spectra respectively. Substituting these into the radial Dirac equation (Eq.(2) ), we get the Schrödinger-like second-order differential equation in terms of the lower and upper spinor components as

$$
\left[-\frac{d^{2}}{d r^{2}}+\frac{C^{2}}{\xi^{2}} V^{2}+2 E_{R} V \mp \frac{C}{\xi} \frac{d V}{d r}-\frac{E_{R}^{2}-1}{\alpha^{2}}\right] \phi^{ \pm}(r)=0,
$$

where the "+" sign belongs to the upper spinor component, while the other sign corresponds to the lower one.

\subsection{Basic Concepts of the Method}

Solutions of the Schrödinger-like second order differential equations play an essential role in studying many important problems of theoretical physics. In this point, the NU method can be used to solve these types equations with an appropriate coordinate transformation $s=s(r)[11]$ :

$$
\psi^{\prime \prime}(s)+\frac{\tilde{\tau}(s)}{\sigma(s)} \psi^{\prime}(s)+\frac{\tilde{\sigma}(s)}{\sigma^{2}(s)} \psi(s)=0
$$

where $\sigma(s)$ and $\tilde{\sigma}(s)$ are polynomials with at most second-degree, and $\tilde{\tau}(s)$ is a firstdegree polynomial. It is of fundamental importance in the study of particular special orthogonal polynomials [13. These polynomials try to reduced Eq.(6) to a simple form by taking $\psi(s)=\phi(s) y(s)$ and choosing an appropriate $\phi(s)$. Consequently, Eq. (6) can be reduced to an equation of hypergeometric type

$$
\sigma(s) y^{\prime \prime}(s)+\tau(s) y^{\prime}(s)+\lambda y(s)=0,
$$

where $\tau(s)=\tilde{\tau}(s)+2 \pi(s)$ (its derivative must be negative) and $\lambda$ is a constant, which is given in the form

$$
\lambda=\lambda_{n}=-n \tau^{\prime}-\frac{n(n-1)}{2} \sigma^{\prime \prime}, \quad(n=0,1,2, \ldots) .
$$

Here, $\lambda$ or $\lambda_{n}$ are obtained from a particular solution of the form $y(s)=y_{n}(s)$ which is a polynomial of degree $n . y_{n}(s)$ is the hypergeometric type function whose polynomial solutions are given by Rodrigues relation

$$
y_{n}(s)=\frac{B_{n}}{\rho(s)} \frac{d^{n}}{d s^{n}}\left[\sigma^{n}(s) \rho(s)\right],
$$

where $B_{n}$ is the normalization constant and the weight function $\rho(s)$ must be satisfied the condition

$$
[\sigma(s) \rho(s)]^{\prime}=\tau(s) \rho(s) .
$$

To determine the weight function given in Eq.(10), we must immediately obtain the polynomial $\pi(s)$ from:

$$
\pi=\frac{\sigma^{\prime}-\tilde{\tau}}{2} \pm \sqrt{\left(\frac{\sigma^{\prime}-\tilde{\tau}}{2}\right)^{2}-\tilde{\sigma}+k \sigma}
$$


Systematical Approach to the Exact Solution of the Dirac Equation...

In principle, the expression under the square root sign in Eq.(11) can be arranged as the square of a polynomial. This is possible only if its discriminant is zero. In this case, it is obtained an equation for $k$. After solving this equation, the obtained values of $k$ are included to the NU method and here there is a relationship with $\lambda$ of $k$ so that $k=\lambda-\pi^{\prime}(s)$. After this point, an appropriate $\phi(s)$ can be invented from $\phi(s)^{\prime} / \phi(s)=\pi(s) / \sigma(s)$.

\section{Special form of the Woods-Saxon potential}

The interaction between nuclei is commonly described by using a potential that consists of the Coulomb and the nuclear potentials. It is usually taken in the form of the WoodsSaxon potential. Here, we take the following special form for the Woods-Saxon potential which is specified by "q" parameter

$$
V(r)=-\frac{q V_{0}}{q+e^{\left(\frac{r-R_{0}}{b}\right)}}
$$

where $V_{0}$ is the potential depth, $R_{0}$ is the width of the potential, $b$ is thickness of the surface which is usually adjusted to the experimental values of ionization energies and $q$ is a real positive parameter which is responsible for the specification of the Woods-Saxon potential. After substituting the potential into Eq.(5), we obtain an equation for the upper spinor component

$$
\begin{gathered}
{\left[-\frac{d^{2}}{d r^{2}}+\frac{C^{2}}{\xi^{2}}\left(\frac{q V_{0}}{q+e^{\left(\frac{r-R_{0}}{b}\right)}}\right)^{2}-\frac{2 q E_{R} V_{0}}{q+e^{\left(\frac{r-R_{0}}{b}\right)}}-\frac{q C V_{0}}{\xi b} \frac{e^{\left(\frac{r-R_{0}}{b}\right)}}{\left(q+e^{\left(\frac{r-R_{0}}{b}\right)}\right)^{2}}-\frac{E_{R}^{2}-1}{\alpha^{2}}\right] \phi^{+}(r)=0,} \\
{\left[-\frac{d^{2}}{d r^{2}}+\frac{q C V_{0}}{\xi b} \frac{\frac{q C V_{0} b}{\xi}-e^{\left(\frac{r-R_{0}}{b}\right)}}{\left(q+e^{\left(\frac{r-R_{0}}{b}\right)}\right)^{2}}-\frac{2 q E_{R} V_{0}}{\left.q+e^{\left(\frac{r-R_{0}}{b}\right)}-\frac{E_{R}^{2}-1}{\alpha^{2}}\right] \phi^{+}(r)=0 .}, \quad(13)\right.}
\end{gathered}
$$

In order to apply the NU-method, we rewrite Eq.(13) by using a new variable of the form $s=-e^{-\left(\frac{r-R_{0}}{b}\right)}$,

$$
\left[-\frac{s}{b} \frac{d}{d s}\left(\frac{s}{b} \frac{d}{d s}\right)+\frac{q^{2} C^{2} V_{0}^{2}}{\xi^{2}}\left(\frac{s}{1-q s}\right)^{2}+\frac{2 q s E_{R} V_{0}}{1-q s}+\frac{q C V_{0}}{\xi b} \frac{s}{(1-q s)^{2}}-\frac{E_{R}^{2}-1}{\alpha^{2}}\right] \phi^{+}(s)=0 \text {. }
$$

By introducing the following dimensionless parameters

$$
\varepsilon=\left(\frac{E_{R}^{2}-1}{\alpha^{2}}\right) b^{2}, \quad \beta=2 q E_{R} V_{0} b^{2}, \quad \gamma=\frac{q C V_{0} b}{\xi},
$$

we reach the following hypergeometric type equation defined in Eq.(6)

$\frac{d^{2} \phi^{+}(s)}{d s^{2}}+\frac{1-q s}{s(1-q s)} \frac{d \phi^{+}(s)}{d s}+\frac{1}{s^{2}(1-q s)^{2}} \times\left[\left(q^{2} \varepsilon+q \beta-\gamma^{2}\right) s^{2}-(2 q \varepsilon+\beta+\gamma) s+\varepsilon\right] \phi^{+}(s)=0$.

After comparing Eq.(16) with Eq.(6), we obtain the corresponding polynomials:

$$
\tilde{\tau}(s)=1-q s, \quad \sigma(s)=s(1-q s), \quad \tilde{\sigma}(s)=\left(q^{2} \varepsilon+q \beta-\gamma^{2}\right) s^{2}-(2 q \varepsilon+\beta+\gamma) s+\varepsilon .
$$


Systematical Approach to the Exact Solution of the Dirac Equation...

Substituting these polynomials into Eq.(11), we organize the polynomial $\pi(s)$ as follows $\pi(s)=-\frac{q s}{2} \pm \frac{1}{2} \sqrt{\left(q^{2}-4\left(q^{2} \varepsilon+q \beta-\gamma^{2}\right)-4 q k\right) s^{2}+4(2 \varepsilon q+\beta+\gamma+k) s-4 \varepsilon}$,

with $\sigma^{\prime}(s)=1-2 q s$. It is taken into consideration that the discriminant of the second order equation under the square root sign has to be zero. Hence, the expected roots are obtained as $k_{ \pm}=(-\beta-\gamma \pm i(q+2 \gamma) \sqrt{\varepsilon})$. In this case, substituting these values for each $k$ into Eq.(18), the possible solutions are obtained for $\pi(s)$

$$
\pi(s)=-\frac{q s}{2} \pm \frac{1}{2}\left\{\begin{array}{c}
(q+2 \gamma-2 i q \sqrt{\varepsilon}) s+2 i \sqrt{\varepsilon} \\
\text { for } k_{+}=-\beta-\gamma+i(q+2 \gamma) \sqrt{\varepsilon} \\
(q+2 \gamma+2 i q \sqrt{\varepsilon}) s-2 i \sqrt{\varepsilon} \\
\text { for } k_{-}=-\beta-\gamma-i(q+2 \gamma) \sqrt{\varepsilon}
\end{array}\right.
$$

From the four possible forms of the polynomial $\pi(s)$, we take a certain one which is the derivative of $\tau(s)$ has a negative value. Therefore, the function $\tau(s)$ satisfies the following equalities:

$$
\begin{aligned}
& \tau(s)=1+2 i \sqrt{\varepsilon}-s(3 q+2 \gamma+2 i q \sqrt{\varepsilon}), \\
& \tau^{\prime}(s)=-(3 q+2 \gamma+2 i q \sqrt{\varepsilon}) .
\end{aligned}
$$

In the present case

$$
\pi(s)=-\frac{q s}{2}-\frac{1}{2}[(q+2 \gamma+2 i q \sqrt{\varepsilon}) s-2 i q \sqrt{\varepsilon}] .
$$

From $k=\lambda-\pi^{\prime}(s)$ and also Eq.(8), we obtain respectively:

$$
\begin{aligned}
& \lambda=-\beta-2 \gamma-q-2 i(\gamma+q) \sqrt{\varepsilon} \\
& \lambda=\lambda_{n}=n^{2} q+2 n q+2 n \gamma+2 n i q \sqrt{\varepsilon} .
\end{aligned}
$$

After having the comparison of Eq.(22) and Eq.(23) and substituting the values of $\varepsilon$ and $\beta$, we can immediately obtain the $\kappa$-independent relativistic energy eigenvalues $E_{R n q}$ of the Dirac particle as follows

$$
\begin{aligned}
E_{R n q}^{ \pm}=-\left[2 b\left(T^{2}+b^{2} q^{2} V_{0}^{2} \alpha^{2}\right)\right]^{-1}\{ & (1+n) q V_{0} b(T+\gamma) \alpha^{2} \pm\left[(1+n)^{2}(T+\gamma)^{2} q^{2} V_{0} b^{2}\right. \\
& \left.\left.-\left(T^{2}+b^{2} q^{2} V_{0}^{2} \alpha^{2}\right)\left((1+n)^{2}(T+\gamma)^{2}-4 b^{2} T^{2}\right)\right]^{1 / 2}\right\},
\end{aligned}
$$

where $T=(1+n) q+\gamma$. To have a physical result, the expression under the square root must be positive. $n$ is a positive integer defined in the interval of $n_{\max } \geq n \geq 0$ and is called the radial quantum number.

By interesting with Eq.(13), we can easily show that, in the non-relativistic limit $\alpha \rightarrow 0$, the relativistic energy is a limit of the non-relativistic energy, $E_{R} \approx 1+\alpha^{2} E_{N R}$, where $E_{N R}$ is the non-relativistic energy. The wave equation is reduced to the following form, choosing $q=1$ :

$$
\left[-\frac{d^{2}}{d r^{2}}+\frac{\gamma}{b^{2}} \frac{\gamma-e^{\left(\frac{r-R_{0}}{b}\right)}}{\left(1+e^{\left(\frac{r-R_{0}}{b}\right)}\right)^{2}}-\frac{2 V_{0}}{1+e^{\left(\frac{r-R_{0}}{b}\right)}}-2 E_{N R}\right] \phi^{+}(r)=0
$$


To obtain a more suitable case, we can use the following form after taking $\gamma=-1$,

$$
\left[-\frac{d^{2}}{d r^{2}}-2 \frac{V_{0}-1 / 2 b^{2}}{1+e^{\left(\frac{r-R_{0}}{b}\right)}}-2 E_{N R}\right] \phi^{+}(r)=0
$$

which is in the form of the Schrödinger equation for the new type s-wave non-relativistic Woods-Saxon potential. The corresponding energy spectrum has already been given in Ref. [14 by using the hypergeometric functions, and also repeated by means of the NU method in Ref. [15] as:

$$
E_{N R}=-\frac{1}{2}\left[\frac{b\left(V_{0}-1 / 2 b^{2}\right)}{n+1}+\frac{n+1}{2 b}\right]^{2},
$$

where the transformation $V_{0} \rightarrow V_{0}-1 / 2 b^{2}$ is applied for the convenience and the index $n$ relates to the radial quantum number $(n=0,1,2,3, \ldots)$. In order to obtain the relativistic energy spectrum directly, considering the relativistic Eq.(13) and the nonrelativistic Eq.(27) for the case of $\gamma=-1$, we can propose the relevant parameter map:

$$
\begin{aligned}
& b \rightarrow b, \quad R_{0} \rightarrow R_{0}, \\
& V_{0}-1 / 2 b^{2} \rightarrow E_{R} V_{0}-1 / 2 b^{2}, \\
& E_{N R} \rightarrow\left(E_{R}^{2}-1\right) / 2 \alpha^{2} .
\end{aligned}
$$

Using the map between the parameters of the two equations, the resulting upper relativistic energy spectrum is found as follows:

$E_{R}^{+}=\frac{-V_{0} b \alpha^{2}\left((n+1)^{2}-1\right)+\sqrt{4 b^{2}(n+1)^{4}+(n+1)^{2} \alpha^{2}\left[4 b^{4} V_{0}^{2}-\left((n+1)^{2}-1\right)^{2}\right]}}{2 b\left((n+1)^{2}+b^{2} V_{0}^{2} \alpha^{2}\right)}$.

It can be easily seen that the relativistic energy spectrum in Eq.(24) gives the same result as Eq.(29) but it can be used only for the s-states. In addition, Eq.(24) indicates that one deals with a family of the Woods-Saxon potential and the relativistic energy spectrum will be also used to describe the single-particle motion in nuclei. Therefore, Eq.(24) can also give the solution of the relativistic Dirac-Woods-Saxon problem with a general value of $\gamma$ for $q=1$.

Let us now find the corresponding wave functions. According to the NUmethod, the polynomial solutions of the hypergeometric function $y(s)$ depend on the determination of weight function $\rho(s)$ satisfying the differential equation $[\sigma(s) \rho(s)]^{\prime}=$ $\tau(s) \rho(s)$. Thus, $\rho(s)$ is calculated as

$$
\rho(s)=(1-q s)^{\nu} s^{2 i \sqrt{\varepsilon}},
$$

where $\nu=1+\frac{2 \gamma}{q}$. Substituting into the Rodrigues relation given in Eq.(9), the wave functions are obtained in the following form

$$
y_{n q}(s)=A_{n}(1-q s)^{-\nu} s^{-2 i \sqrt{\varepsilon}} \frac{d^{n}}{d s^{n}}\left[(1-q s)^{n+\nu} s^{n+2 i \sqrt{\varepsilon}}\right],
$$

where $A_{n}$ is the normalization constant. Taking $q=1$, the polynomial solutions of $y_{n}(s)$ are expressed in terms of the Jacobi Polynomials, which is one of the orthogonal 
Systematical Approach to the Exact Solution of the Dirac Equation...

polynomials. In this case, the weight function is $(1-s)^{\nu} s^{2 i \sqrt{\varepsilon}}$ and Eq.(131) is reduced to $\sim P_{n}^{(2 i \sqrt{\varepsilon}, \nu)}(1-2 s)$ [13. After substituting $\pi(s)$ and $\sigma(s)$ into the expression $\phi(s)^{\prime} / \phi(s)=\pi(s) / \sigma(s)$, the other part of the wave function is found as

$$
\phi(s)=(1-q s)^{\nu-\gamma / q} s^{i \sqrt{\varepsilon}} .
$$

We write the upper spinor component in terms of the Jacobi polynomials

$$
\phi_{n}^{+}(s)=B_{n} s^{i \sqrt{\varepsilon}}(1-s)^{\nu-\gamma} P_{n}^{(2 i \sqrt{\varepsilon}, \nu)}(1-2 s),
$$

where $B_{n}$ is a normalization constant. The lower component of the spinor wave function can also be obtained by substituting Eq.(33) into Eq.(3). We should then solve the following equation

$$
\phi_{n}^{-}(s)=\frac{\alpha}{C+E_{R n}^{ \pm}}\left[-\xi+\frac{C}{\xi} V(s)-\frac{s}{b} \frac{d}{d s}\right] \phi_{n}^{+}(s) .
$$

where $E_{R n}^{ \pm} \neq C$. This is possible if a new variable is introduced as $x=1-2 s$. Now, the equation of the lower spinor component has been transformed into the following form

$$
\phi_{n}^{-}(s)=\frac{\alpha}{C+E_{R n}^{ \pm}}\left[-\xi+\frac{V_{0} C(1-x)}{\xi(1+x)}+\frac{(1-x)}{b} \frac{d}{d x}\right] \phi_{n}^{+}(s),
$$

with

$$
\phi_{n}^{+}(s)=C_{n}(1-x)^{i \sqrt{\varepsilon}}(1+x)^{\nu-\gamma} P_{n}^{(2 i \sqrt{\varepsilon}, \nu)}(x),
$$

where $C_{n}$ is the normalization constant and its value is equal to $B_{n} 2^{\gamma-\nu-i \sqrt{\varepsilon}}$. If the following recursion relations and the differential formula satisfied by the Jacobi polynomials [16] are included to the solution

$$
\begin{aligned}
& (1+x) P_{n}^{(\mu, \varrho)}(x)=\frac{2}{2 n+\mu+\varrho+1}\left[(n+\varrho) P_{n}^{(\mu, \varrho-1)}(x)+(n+1) P_{n+1}^{(\mu, \varrho-1)}(x)\right], \\
& (1-x) P_{n}^{(\mu, \varrho)}(x)=\frac{2}{2 n+\mu+\varrho+1}\left[(n+\mu) P_{n}^{(\mu-1, \varrho)}(x)-(n+1) P_{n+1}^{(\mu-1, \varrho)}(x)\right], \\
& \left(1-x^{2}\right) \frac{d P_{n}^{(\mu, \varrho)}}{d x}(x)=-n\left(x+\frac{\varrho-\mu}{2 n+\mu+\varrho}\right) P_{n}^{(\mu, \varrho)}(x)+2 \frac{(n+\mu)(n+\varrho)}{2 n+\mu+\varrho} P_{n-1}^{(\mu, \varrho)}(x), \\
& P_{n}^{(\mu, \varrho)}(x)=\frac{n+\mu+\varrho+1}{2 n+\mu+\varrho+1} P_{n}^{(\mu, \varrho+1)}(x)+\frac{n+\mu}{2 n+\mu+\varrho+1} P_{n-1}^{(\mu, \varrho+1)}(x),
\end{aligned}
$$

we obtained the lower spinor component in terms of the Jacobi polynomials as a function of $s(r)$

$$
\begin{array}{r}
\phi_{n}^{-}(s)=\frac{B_{n}}{2 n+\mu+\varrho+1} \frac{\alpha}{C+E_{R n}^{ \pm}}(1-s)^{\gamma} s^{i \sqrt{\varepsilon}}\left\{( L + n / b ) \left[(n+\mu) P_{n}^{(2 i \sqrt{\varepsilon}-1, \nu)}(1-2 s)\right.\right. \\
\left.-(n+1) P_{n+1}^{(2 i \sqrt{\varepsilon}-1, \nu)}(1-2 s)\right]+2(n+\varrho)(2 n+\mu+\varrho+1-M) P_{n}^{(2 i \sqrt{\varepsilon}, \nu-1)}(1-2 s) \\
\left.-(1 / 2 b+(n+2 b)(2 n+\mu+\varrho+1) / 2) P_{n}^{(2 i \sqrt{\varepsilon}, \nu)}(1-2 s)\right\},
\end{array}
$$

where $L=C V_{0} / \xi+(1+\gamma) / b$ and $M=\xi+i \sqrt{\varepsilon} / b$. 


\section{Conclusion}

We have solved the Dirac equation for the special form of the Woods-Saxon potential following a formalism introduced by Alhaidari. The NU method is used to obtain a systematical solution in the Dirac-Woods-axon problem. The energy spectrum of the bound states is analytically obtained and two-component spinor eigenfunctions are written in terms of the Jacobi polynomials. It is seen that the energy eigenvalues are a function of the parameter $\mathrm{q}$ and the solution space splits into two distinct subspaces. We have seen that the non-relativistic limit of the Dirac equation can be obtained easily. We can also say that the exact results obtained for a special form of the Woods-Saxon potential give us some interesting applications in the various quantum mechanical studies and the relativistic nuclear scattering problems.

\section{Acknowledgements}

The authors are indebted to A. D. Alhaidari for the useful comments on the original version of the manuscript. This research was partially supported by the Scientific and Technological Research Council of Turkey. 
[1] A. Bohr and B.R. Mottelson, Nuclear Structure, Vol. I, Section 2.4 (Benjamin/Cummmings, New York, 1969);

W. R. Pierson et al., Phys. Rev. B 133, 384 (1964);

K. Clemenger, Phys. Rev. B 32, 1359 (1985).

[2] A. A. Ogloblin et al., Phys. Rev. C 62, 044601 (2000).

[3] J. Bentez, R. P. Martinezy-Romero, H. N. Nunez-Yepez, and A. L. Salas-Brito, Phys. Rev. Lett. 64, 1643 (1990).

[4] P. Rozmej and R. Arvieu, J. Phys. A 32, 5367 (1999).

[5] J. N. Ginocchio, Phys. Rev. C 69, 034318 (2004).

[6] R. Lisboa, M. Malheiro, A. S. de Castro, P. Alberto and M. Fiolhais, Phys. Rev. C 69, 024319 (2004).

[7] P. Kennedy, J. Physics A: Math. Gen. 35, 689 (2002);

G. J. You, F. X. Zheng, and X. F. Xin, Phys. Rev. A 66, 062105 (2002);

A. D. Alhaidari, J. Physics A 37.22, 5805 (2004).

[8] A.D. Alhaidari, Int. J. Mod. Phys. A 18, 4955 (2003).

[9] A. D. Alhaidari, Phys. Rev. Lett. 87, 210405 (2001); ibid. 88, 189901 (2002);

A. D. Alhaidari, J. Phys. A 34, 9827 (2001).

[10] J. Y. Guo, J. Meng, and F. X. Xu, Chin. Phys. Lett. 20, 602 (2003).

[11] A. F. Nikiforov, V. B. Uvarov, Special Functions of Mathematical Physics, (Birkhauser, Basel, 1988).

[12] C. Berkdemir, A. Berkdemir and R. Sever, Phys. Rev. C 72, 027001 (2005);

H. Fakhri, and J. Sadeghi, Mod. Phys. Lett. A 29, 615 (2004).

[13] G. Szego, Orthogonal Polynomials, (American Mathematical Society, New York, 1939).

[14] S. Flügge, Practical Quantum Mechanics I, (Springer-Verlag, Berlin, 1974).

[15] A. Berkdemir, C. Berkdemir and R. Sever, accepted for publication in Modern Physics Letters A, (2006); Preprint, quant-ph/0410153.

[16] W. Magnus, F. Oberhettinger, and R.P. Soni, Formulas and Theorems for the Special Functions of Mathematical Physics, (Springer-Verlag, New York, 1966);

M. Abramowitz an I. A. Stegun (eds.), Handbook of Mathematical Functions, (Dover, New York, 1964). 